\title{
Ipragliflozin Reduces Epicardial Fat Accumulation in Non-Obese Type 2 Diabetic Patients with Visceral Obesity: A Pilot Study
}

\author{
Tatsuya Fukuda - Ryotaro Bouchi (D) Masahiro Terashima • Yuriko Sasahara • \\ Masahiro Asakawa · Takato Takeuchi · Yujiro Nakano • \\ Masanori Murakami · Isao Minami · Hajime Izumiyama - Koshi Hashimoto • \\ Takanobu Yoshimoto · Yoshihiro Ogawa
}

Received: May 9, 2017 / Published online: June 14, 2017

(C) The Author(s) 2017. This article is an open access publication

\section{ABSTRACT}

Introduction: Epicardial fat (EF) was reported to be independently associated with cardiovascular disease regardless of obesity. We have previously reported that a sodium-glucose co-transporter-2 (SGLT2) inhibitor, luseogliflozin, reduces the EF volume (EFV) in parallel

Enhanced content To view enhanced content for this article go to http://www.medengine.com/Redeem/ 5B98F06039A57F09.

T. Fukuda $\cdot$ R. Bouchi $(\bowtie) \cdot$ Y. Sasahara

M. Asakawa · T. Takeuchi · Y. Nakano .

M. Murakami · I. Minami · H. Izumiyama .

K. Hashimoto · T. Yoshimoto · Y. Ogawa

Department of Molecular Endocrinology and

Metabolism, Graduate School of Medical and Dental

Sciences, Tokyo Medical and Dental University,

Tokyo, Japan

e-mail: bouchi.mem@tmd.ac.jp

M. Terashima

Cardiovascular Imaging Clinic, Tokyo, Japan

H. Izumiyama

Center for Medical Welfare and Liaison Services,

Tokyo Medical and Dental University, Tokyo, Japan

K. Hashimoto

Department of Preemptive Medicine and

Metabolism, Graduate School of Medical and Dental

Sciences, Tokyo Medical and Dental University,

Tokyo, Japan

Y. Ogawa

CREST, Japan Agency for Medical Research and

Development, Tokyo, Japan with the reduction of body weight in obese patients (BMI $\geq 25 \mathrm{~kg} / \mathrm{m}^{2}$ ) with type 2 diabetes. However, it is unknown whether SGLT2 inhibitors could reduce EFV in non-obese patients (BMI $<25 \mathrm{~kg} / \mathrm{m}^{2}$ ) with type 2 diabetes. Therefore, we evaluated the effect of SGLT2 inhibitors on the EFV in non-obese type 2 diabetic patients with visceral obesity in this pilot study.

Methods: Nine of type 2 diabetic patients (mean age $66 \pm 8$ years; $33 \%$ female) with $\mathrm{HbA}_{1 \mathrm{c}} 6.5-9.0 \%$, body mass index (BMI, $\mathrm{kg} / \mathrm{m}^{2}$ ) $<25.0$, and visceral fat area (VFA, $\left.\mathrm{cm}^{2}\right) \geq 100$ were enrolled. Participants were administered ipragliflozin $50 \mathrm{mg}$ daily. EFV [median (interquartile range), $\mathrm{cm}^{3}$ ] was measured by magnetic resonance imaging. Primary endpoint was the change in EFV at 12 weeks. VFA and liver attenuation index (LAI), skeletal muscle index (SMI), and body fat (\%) were also assessed at baseline and at 12 weeks.

Results: The EFV was significantly reduced from $102(79-126) \mathrm{cm}^{3}$ to $89(66-109) \mathrm{cm}^{3}$ by ipraglifrozin $(p=0.008)$. The body weight, BMI, $\mathrm{HbA}_{1 \mathrm{c}}$, fasting plasma glucose, insulin, homeostasis model assessment-insulin resistance, triglycerides, leptin, body fat, android, gynoid, and VFA were significantly reduced and high-density lipoprotein cholesterol was significantly increased by ipraglifrozin at 12 weeks, whereas SFA and LAI were unchanged. The change in EFV was significantly correlated with the change in BMI. 
Conclusions: A12-week intervention of ipragliflozin reduced the EFV in non-obese type 2 diabetic patients with visceral adiposity.

Clinical Trial Registration: UMIN Clinical Trial Registry: UMIN000019071.

Funding: Astellas Pharma Inc. and the Grants-in-Aid for Scientific Research from the Ministry of Education, Culture, Sports, Science and Technology of Japan.

Keywords: Epicardial fat; Ipragliflozin; Normal weight; Sodium-glucose co-transporter-2 inhibitors; Type 2 diabetes; Visceral obesity

\section{INTRODUCTION}

It is well established that obesity is associated with insulin resistance and increases the risk of type 2 diabetes and cardiovascular disease (CVD) [1-3]. As for body fat distribution, visceral fat (VF) accumulation has been more strongly associated with cardiometabolic risks than the body mass index (BMI), waist circumference, and abdominal subcutaneous fat accumulation [4, 5]. Indeed, we have previously reported that VF accumulation is independently associated with the progression of arterial stiffness assessed by brachial-ankle pulse wave velocity (baPWV) in non-obese (BMI $<25 \mathrm{~kg} /$ $\mathrm{m}^{2}$ ) patients with type 2 diabetes [6]. Consequently, VF accumulation is thought to be a therapeutic target in non-obese patients with type 2 diabetes as well as obese patients. In addition to VF, epicardial fat (EF), a fat deposit located between the heart and the pericardium, is one of the phenotypes of ectopic fat accumulation $[7,8]$. EF has the same embryological origin as VF [9] and is correlated with the degree of obesity (BMI) [10]. It has also been reported that $\mathrm{EF}$ is associated with cardiometabolic risks including insulin resistance $[11,12]$, produces proinflammatory cytokines as well as VF [7], and patients with high EF are at an increased risk for both fatal and nonfatal coronary events, independently of traditional cardiovascular risk factors [13], suggesting that EF may be implicated in the pathogenesis of atherosclerosis, especially coronary artery disease. Among non-obese patients who were suspected of coronary artery disease, EF was shown to be associated with coronary plaques $[14,15]$. Given these findings, it is possible that EF may play an important role in developing an unfavorable cardiovascular risk profile and CVD, regardless of obesity.

Sodium-glucose co-transporter-2 (SGLT2) inhibitors are a new group of oral medications for the treatment of type 2 diabetes through the suppression of reabsorption of glucose in the proximal tubule. Recently, the EMPA-REG outcome study demonstrated favorable cardiovascular outcomes in diabetic patients treated with empagliflozin [16]. It has been suggested that the outcomes are caused by not only the improvement of glycemic control but also the multifactorial changes in cardiovascular risk profiles including a decline in weight, blood pressure, albuminuria, and visceral fat, and an improvement of insulin sensitivity [17-20]. Although the precise mechanism of the favorable effects of SGLT2 inhibitors on CVD is still fully unknown, it is expected that the reduction of $\mathrm{EF}$ volume (EFV) in addition to the reduction of either body weight or VF may be associated with the improvement of cardiovascular outcomes. We previously reported that an SGLT2 inhibitor, luseogliflozin, could reduce the EFV in parallel with the improvement of systemic microinflammation and the reduction of body weight in obese (BMI $\geq 25 \mathrm{~kg} / \mathrm{m}^{2}$ ) patients with type 2 diabetes [21]. However, it is unknown whether SGLT2 inhibitors reduce the EFV in non-obese (BMI $<25 \mathrm{~kg} / \mathrm{m}^{2}$ ) patients with type 2 diabetes. Among SGLT2 inhibitors, ipraglifrozin has been reported to sufficiently improve hyperglycemia and its safety was unaffected by BMI in a pooled analysis of Japanese patients with type 2 diabetes [22]. Therefore, we conducted a pilot study to investigate the effect of ipragliflozin on the EFV in non-obese Japanese patients with type 2 diabetes.

\section{METHODS}

\section{Study Design}

Patients with type 2 diabetes who were nonobese (BMI $<25.0 \mathrm{~kg} / \mathrm{m}^{2}$ ) but had an increased 
visceral adiposity (a visceral fat area (VFA) $\geq 100 \mathrm{~cm}^{2}$ ] were enrolled in this 12 -week single-arm pilot study from 15 October 2015 to 31 August 2016. All procedures followed were in accordance with the ethical standards of the responsible committee on human experimentation (institutional and national) and with the Helsinki Declaration of 1964, as revised in 2013. Informed consent was obtained from all patients for being included in the study. This study has been approved by the ethical committee of Tokyo Medical and Dental University (No. 2109). This study was registered at the UMIN Clinical Trial Registry (UMIN000019071).

\section{Subjects}

Patients with type 2 diabetes aged older than 20 years who regularly visited the Tokyo Medical and Dental University Hospital were screened in this study. Patients who met the following criteria at the start of the treatment were eligible for the study: (1) they were diagnosed as having type 2 diabetes according to the criteria of the Japan Diabetes Society (JDS) [23], (2) they had a BMI less than $25 \mathrm{~kg} / \mathrm{m}^{2}$, (3) their $\mathrm{HbA}_{1 \mathrm{c}}$ levels were between $6.5 \%$ and $9.0 \%$, (4) VFA was equal to or greater than $100 \mathrm{~cm}^{2}$. Patients who met any of the following criteria were excluded from the study: (1) any history of taking SGLT2 inhibitors, (2) insulin treatment, (3) active infection, malignancy, congestive heart failure, severe liver, and renal diseases, (4) severe diabetic retinopathy, (5) pregnant women, (6) any history of acute coronary syndrome or stroke within 3 months prior to the enrollment, (7) patients whose treatment for diabetes was changed 3 months prior to baseline evaluation, (8) those who had contraindications for magnetic resonance imaging (MRI). Written informed consent was obtained from all patients before the enrollment.

\section{Intervention}

The patients were administered $50 \mathrm{mg}$ of ipragliflozin once daily after breakfast. During the study period of 12 weeks, antihyperglycemic agents were unchanged, except when unacceptable hyperglycemia, hypoglycemia, or adverse events occurred. The diet and exercise therapy were kept unchanged during the study.

\section{Clinical and Biochemical Analysis}

The BMI was calculated as the weight divided by the square of the height $\left(\mathrm{kg} / \mathrm{m}^{2}\right)$. The systolic and diastolic blood pressures (SBP and DBP) were measured in a sitting position after at least $5 \mathrm{~min}$ rest, using an electronic sphygmomanometer (ES-H55, Terumo Inc., Tokyo, Japan). Blood samples were collected at an overnight fasting state. Routine tests included alanine transaminase (ALT), aspartate transaminase (AST), gamma-glutamyl transpeptidase $(\gamma-G T P)$, high-density lipoprotein (HDL) and low-density lipoprotein (LDL) cholesterol, triglycerides, uric acid, C-reactive protein, hemoglobin, and hematocrit. $\mathrm{HbA}_{1 \mathrm{c}}$ was measured using the latex agglutination method. The insulin sensitivity was assessed by a homeostasis model assessment as an index of insulin resistance (HOMA-IR) in patients whose fasting plasma glucose levels were less than $7.8 \mathrm{mmol} / \mathrm{l}$. Urinary albumin and creatinine excretion were measured in a spot urine collection by a turbidimetric immunoassay and enzymatic method. The ratio [albumin-to-creatinine ratio (ACR), $\mathrm{mg} / \mathrm{g}$ ] was used for the assessment of albuminuria. The GFR was calculated using the equation for the Japanese [24]. Adiponectin, leptin, and interleukin 6 (IL-6) were measured using latex turbidimetric immunoassay, double-antibody radioimmunoassay, and chemiluminescent enzyme immunoassay, respectively.

\section{Quantification of Body Composition Measured by DXA and Abdominal CT}

The whole body DXA (Lunar iDXA, GE Healthcare, Madison, WI) was used for the assessment of the regional fat and fat-free mass as described previously [25]. The skeletal muscle index (SMI) was calculated as follows: the appendicular nonfat mass divided by the square of the height $(\mathrm{kg} /$ $\mathrm{m}^{2}$ ). The body fat (\%) was calculated as the whole body fat mass $(\mathrm{kg})$ divided by the weight $(\mathrm{kg})$. The abdominal computed tomography 
examination (Aquilion PRIME, Toshiba Medical Systems, Tochigi, Japan) was used for the assessment of VFA, subcutaneous fat area (SFA), and liver attenuation index (LAI) as described previously $[25,26]$. The LAI was defined as the average attenuation value of the liver (eight points) divided by the average attenuation value of the spleen (three points).

\section{Quantification of Epicardial Fat Volume Measured by MRI}

The EFV was measured using a 1.5-T MRI system (Achieva Dual, Philips Healthcare, Netherlands or Titan, Toshiba Medical Systems, Japan) with a 32-channel commercially available cardiac coil and was assessed using a modified fat excitation based on the whole-heart coronary MR angiography sequence [27]. A patient-specific acquisition window was set during either systole or diastole, depending on the phase of minimal motion of the right coronary artery. Typical parameters of the sequence included repetition time, $3.2 \mathrm{~ms}$; echo time, $1.6 \mathrm{~ms}$; flip angle, $15^{\circ}$; sensitivity-encoding factor, 2.5; field of view, $330 \times 330 \times 128 \mathrm{~mm}$; acquisition matrix, $248 \times 238$; acquired spatial resolution, $1.3 \times 1.4 \times 1.6 \mathrm{~mm}$, and reconstructed voxel size, $0.64 \times 0.64 \times 0.8 \mathrm{~mm}$. All MR images were transferred to a workstation (Ziostation 2, Japan). Semiautomatic analyses of EFV were performed by an experienced operator blinded to the clinical information.

\section{Study Endpoints}

The primary endpoint of this study was the change in EFV during the follow-up of 12 weeks. The secondary analyses included the changes in the following variables at 12 weeks: VFA, SFA, SMI, LAI, $\mathrm{HbA}_{1 \mathrm{c}}$, HOMA-IR, blood pressure, lipid profile, ACR, C-reactive protein (CRP), adiponectin, leptin, and IL-6.

\section{Statistical Analysis}

Statistical analysis was carried out using an IBM SPSS version 21.0 statistical package (IBM Corp. Released 2012. IBM SPSS Statistics for Windows, Version 21.0. Armonk, NY: IBM Corp.). Data are presented as the mean \pm standard deviation (SD), median with interquartile range (IQR), or percentage as appropriate according to the data distribution. Pearson product-moment correlation analysis was used to investigate the correlation of EFV with the markers for body composition and cardiometabolic risks. The primary endpoint was analyzed using the Wilcoxon signed-rank test. The secondary endpoints were also analyzed by paired $t$ test or Wilcoxon signed-rank test. $P$ values less than 0.05 were considered to be statistically significant.

\section{RESULTS}

\section{Demographics}

Ten patients were recruited and informed consent was obtained from all subjects. At baseline, one patient was excluded because the VFA level was less than $100 \mathrm{~cm}^{2}$ and finally nine patients [age $66 \pm 8$ (range 47-74) years, female 33\%] completed the study. Table 1 presents the baseline demographic data and Table 2 shows the medications at baseline.

\section{Efficacy and Safety}

As shown in Fig. 1, the EFV was significantly reduced by the administration of ipraglifrozin from $102(79-126) \mathrm{cm}^{3}$ to $89(66-109) \mathrm{cm}^{3}$ $(p=0.008)$. The body weight, BMI, plasma glucose, insulin, HOMA-IR, and uric acid levels were significantly decreased and the HDL cholesterol and hemoglobin levels were significantly increased by the ipraglifrozin treatment. The ipraglifrozin did not affect the renal function. Table 3 shows the changes in the body composition before and after the administration of ipraglifrozin. The whole body fat mass, percentage body fat, android, and gynoid were significantly decreased by the ipraglifrozin treatment. In contrast, neither non-fat mass nor SMI was changed at 12 weeks after the administration of ipraglifrozin. In addition, VFA was significantly decreased, whereas SFA was not decreased at 12 weeks after the administration of ipraglifrozin (Fig. 2). When considering the effect of ipraglifrozin on the circulating 
Table 1 Clinical characteristics at baseline and at 12 weeks after the administration of ipragliflozin in patients with type 2 diabetes

\begin{tabular}{|c|c|c|c|}
\hline & Baseline & 12 weeks & $p$ values \\
\hline Age (years) & $66 \pm 8$ & & \\
\hline Gender ( $\%$ female) & 33 & & \\
\hline $\mathrm{SBP}(\mathrm{mmHg})$ & $135 \pm 16$ & $129 \pm 14$ & 0.143 \\
\hline $\mathrm{DBP}(\mathrm{mmHg})$ & $89 \pm 14$ & $84 \pm 10$ & 0.204 \\
\hline Body weight $(\mathrm{kg})$ & $59.3 \pm 7.8$ & $58.1 \pm 7.8$ & 0.011 \\
\hline Body mass index $\left(\mathrm{kg} / \mathrm{m}^{2}\right)$ & $22.6 \pm 1.8$ & $22.1 \pm 1.8$ & 0.011 \\
\hline Waist circumference $(\mathrm{cm})$ & $86 \pm 6$ & $86 \pm 6$ & 0.973 \\
\hline $\mathrm{HbAlc}(\mathrm{mmol} / \mathrm{mol})$ & $55.1 \pm 6.8$ & $50.9 \pm 4.1$ & 0.011 \\
\hline HbAlc (\%) & $7.2 \pm 0.6$ & $6.8 \pm 0.4$ & 0.011 \\
\hline Plasma glucose $(\mathrm{mmol} / \mathrm{l})$ & $7.7(7.1-8.7)$ & $6.9(6.0-7.6)$ & 0.050 \\
\hline Insulin $(\mu \mathrm{U} / \mathrm{ml})$ & $9.1(6.4-12.2)$ & $7.1(5.2-9.8)$ & 0.022 \\
\hline HOMA-IR $(N=7)$ & $2.28(1.44-2.97)$ & $1.84(1.41-2.02)$ & 0.007 \\
\hline Triglycerides $(\mathrm{mmol} / \mathrm{l})$ & $2.49(0.87-2.91)$ & $1.66(0.75-4.04)$ & 0.878 \\
\hline HDL cholesterol $(\mathrm{mmol} / \mathrm{l})$ & $1.43 \pm 0.34$ & $1.54 \pm 0.35$ & 0.042 \\
\hline LDL cholesterol $(\mathrm{mmol} / \mathrm{l})$ & $2.92 \pm 1.03$ & $2.81 \pm 1.08$ & 0.409 \\
\hline $\operatorname{AST}(\mathrm{U} / \mathrm{l})$ & $21(18-54)$ & $29(18-39)$ & 0.878 \\
\hline $\operatorname{ALT}(\mathrm{U} / \mathrm{l})$ & $26(16-55)$ & $25(15-48)$ & 0.476 \\
\hline$\gamma$-GTP $(\mathrm{U} / \mathrm{l})$ & $70(32-149)$ & $69(31-90)$ & 0.173 \\
\hline Estimated GFR $\left(\mathrm{ml} / \mathrm{min} / 1.73 \mathrm{~m}^{2}\right)$ & $79.5 \pm 17.1$ & $77.3 \pm 15.8$ & 0.488 \\
\hline Urinary ACR $(\mathrm{mg} / \mathrm{g})$ & $14(11-17)$ & $15(8-32)$ & 0.510 \\
\hline Uric acid $(\mu \mathrm{mol} / \mathrm{L})$ & $326 \pm 68$ & $281 \pm 70$ & 0.006 \\
\hline Hemoglobin $(\mathrm{g} / \mathrm{dl})$ & $14.9 \pm 1.6$ & $15.7 \pm 1.3$ & 0.019 \\
\hline $\mathrm{CRP}(\mathrm{mg} / \mathrm{l})$ & $0.55(0.33-3.48)$ & $0.40(0.33-1.58)$ & 0.476 \\
\hline
\end{tabular}

$A C R$ albumin-to-creatinine ratio, $A L T$ alanine transaminase, $A S T$ aspartate transaminase, $C R P$ C-reactive protein, $D B P$ diastolic blood pressure, GFR glomerular filtration ratio, GTP glutamyl transpeptidase, $H D L$ high-density lipoprotein, HOMA-IR homeostasis model assessment as an index of insulin resistance, $L D L$ low-density lipoprotein, $S B P$ systolic blood pressure

adipokine levels, the leptin level was significantly decreased by the ipraglifrozin treatment, whereas adiponectin and IL-6 level were unchanged by the ipraglifrozin (Fig. 3). No severe adverse events including severe genitourinary infection and hypoglycemia were observed during the study period.

\section{Correlation of Changes in EFV with Changes in Markers for Body Composition and Cardiometabolic Risks by Ipragliflozin}

Table 4 shows the correlation of the changes in $\mathrm{EFV}$ with the changes in the markers for body 
Table 2 Medications at baseline

\begin{tabular}{lr}
\hline Sulfonylureas (\%) & 0 \\
Biguanides (\%) & 67 \\
Alpha-GIs (\%) & 11 \\
Glinides (\%) & 22 \\
TZDs (\%) & 33 \\
DPP4 inhibitors (\%) & 0 \\
GLP1 receptor agonists (\%) & 33 \\
ARBs (\%) & 22 \\
Calcium channel blockers (\%) & 33 \\
Statins (\%) & 11 \\
Ezetimib (\%) & 11 \\
Antiplatelet agents (\%) &
\end{tabular}

$A R B s$ angiotensin receptor blockers, DPP4 dipeptidyl peptidase-4, GIs glycosidase inhibitors, GLP1 glucagon-like peptide-1, TZDs thiazolidinediones

composition, cardiometabolic risks, and adipokines. The change in EFV was significantly correlated with the changes in both weight and BMI. The changes in hepatic fat (LAI) and visceral fat (VFA) accumulation and muscle mass (SMI) were not correlated with the change in EFV. No significant correlation was observed between EFV and cardiometabolic risk factors and adipokines in this study.

\section{DISCUSSION}

We demonstrate in this single-arm pilot study that an SGLT2 inhibitor, ipragliflozin, significantly reduces EFV in parallel with the reduction of weight and the improvement of glycemic control, lipid profile, and insulin resistance in non-obese type 2 diabetic patients with increased visceral adiposity. We have previously reported that non-obese type 2 diabetic patients with increased visceral adiposity have a high risk for the accumulation of cardiometabolic risk factors and for the prevalent fatty liver disease [26]. We have further demonstrated that high visceral fat with normal weight is strongly associated with the risk for

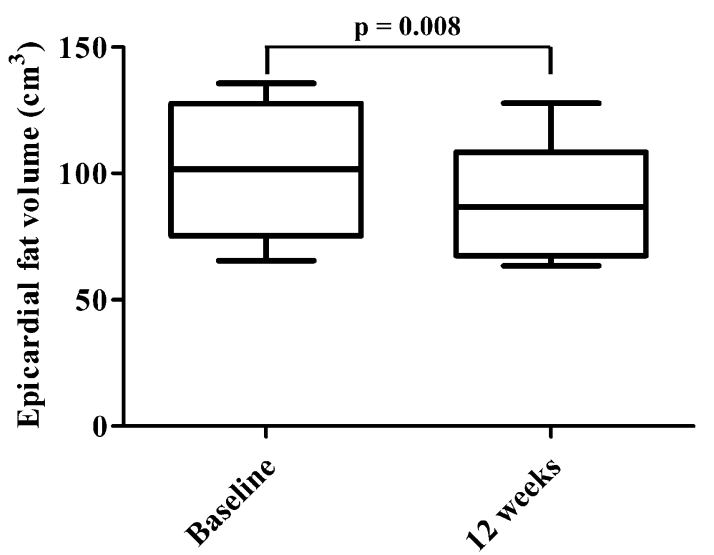

Fig. 1 Epicardial fat (EF) volume at baseline and at 12 weeks after the administration of ipraglifrozin. Data are presented as the median with interquartile range

the progression of arterial stiffness among diabetic population [6]. Given these findings, it seems important to reduce adipose tissue including visceral fat for the prevention of future cardiovascular events even in patients without obesity. To our knowledge, this is the first study to examine the effect of SGLT2 inhibitors on the EFV in non-obese patients with type 2 diabetes.

EF accumulation has recently been reported to be associated with a non-calcified coronary plaque [13, 28, 29] and cardiometabolic risks including insulin resistance $[11,12]$. The Heinz Nixdorf Recall Study, a population-based prospective cohort study of 4093 participants, revealed that $\mathrm{EF}$ accumulation independently increased the risk of both fatal and nonfatal coronary events even after adjustment for traditional cardiovascular risk factors [13]. The study also showed that diabetes is significantly correlated with EFV. Diabetes per se was recently reported to be associated with cardiac structural abnormalities regardless of obesity [30]. In the study, myocardial triglyceride contents measured by the magnetic resonance spectroscopy was significantly higher in nonobese patients with type 2 diabetes compared with non-diabetic subjects. It is therefore conceivable that the EFV could be associated with the risk for CVD in non-obese patients with diabetes. In our study, the administration of ipragliflozin significantly reduced EFV (Fig. 1), 
Table 3 Changes in body composition after the administration of ipragliflozin in patients with type 2 diabetes

\begin{tabular}{|c|c|c|c|}
\hline & Baseline & 12 weeks & $p$ values \\
\hline Body fat (\%) & $31.9 \pm 5.5$ & $31.1 \pm 5.9$ & 0.024 \\
\hline $\begin{array}{l}\text { Non-fat mass } \\
\text { in upper } \\
\text { extremities } \\
(\mathrm{kg})\end{array}$ & $4.7(3.8-5.2)$ & $4.7(3.6-5.1)$ & 0.903 \\
\hline $\begin{array}{l}\text { Non-fat mass } \\
\text { in lower } \\
\text { extremities } \\
(\mathrm{kg})\end{array}$ & $\begin{array}{l}12.4 \\
\quad(11.2-13.8)\end{array}$ & $\begin{array}{l}12.6 \\
\quad(11.4-13.6)\end{array}$ & 0.799 \\
\hline Android $(\mathrm{kg})$ & $2.0(1.7-4.4)$ & $1.8(1.6-2.1)$ & 0.013 \\
\hline Gynoid (kg) & $2.6(2.2-7.2)$ & $2.3(2.0-2.4)$ & 0.009 \\
\hline $\begin{array}{l}\text { Total fat mass } \\
\quad(\mathrm{kg})\end{array}$ & $\begin{array}{l}18.6 \\
\quad(17.3-20.6)\end{array}$ & $\begin{array}{l}18.2 \\
\quad(16.7-19.5)\end{array}$ & 0.004 \\
\hline $\begin{array}{l}\text { Total non-fat } \\
\text { mass }(\mathrm{kg})\end{array}$ & $\begin{array}{l}41.4 \\
\quad(37.2-43.3)\end{array}$ & $\begin{array}{l}41.1 \\
\quad(36.5-43.3)\end{array}$ & 0.241 \\
\hline $\begin{array}{l}\text { Skeletal muscle } \\
\text { index }\end{array}$ & $\begin{array}{l}6.50 \\
\quad(6.19-6.74)\end{array}$ & $\begin{array}{l}6.49 \\
\quad(6.01-6.70)\end{array}$ & 0.386 \\
\hline
\end{tabular}

presumably contributing to the prevention of future CVD even in non-obese patients with diabetes.

Several previous studies have mentioned the potential of exercise and medications to reduce EFV. Kim et al. presented evidence that aerobic exercise training reduces $\mathrm{EF}$ as well as $\mathrm{VF}$ and weight in obese men [31]. In diabetic patients, some antidiabetic medications have been reported to be effective in the reduction of EFV. Sacks et al. showed the possibility that treatment with pioglitazone in type 2 diabetic patients with coronary artery disease reduced proinflammatory cytokines in the EF [32]. In addition, the treatment with sitagliptin was reported to be associated with the reduction of $\mathrm{EF}$ in patients who were taking metformin as monotherapy [33]. The treatment with liraglutide, a glucagon-like peptide- 1 receptor agonist, was also indicated to contribute to the reduction of EF, independently of weight loss [34]. Additionally, we reported that an SGLT2 inhibitor, luseogliflozin, could reduce the EFV in

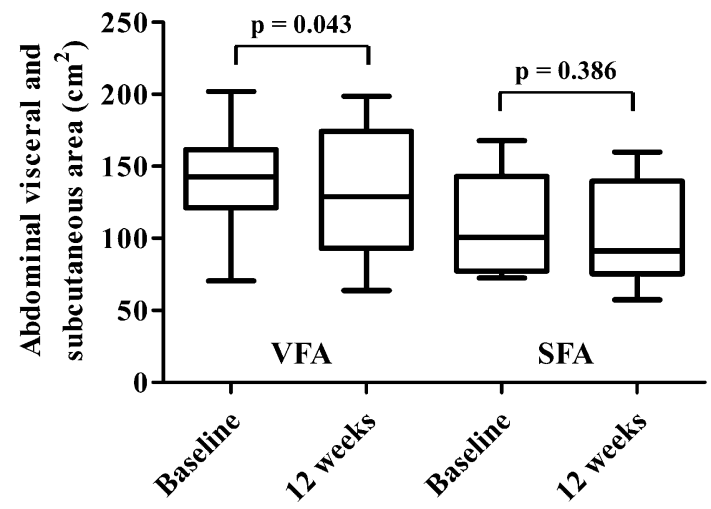

Fig. 2 Abdominal visceral fat area (VFA) and subcutaneous fat area (SFA) at baseline and at 12 weeks after the administration of ipraglifrozin. Data are presented as the median with interquartile range

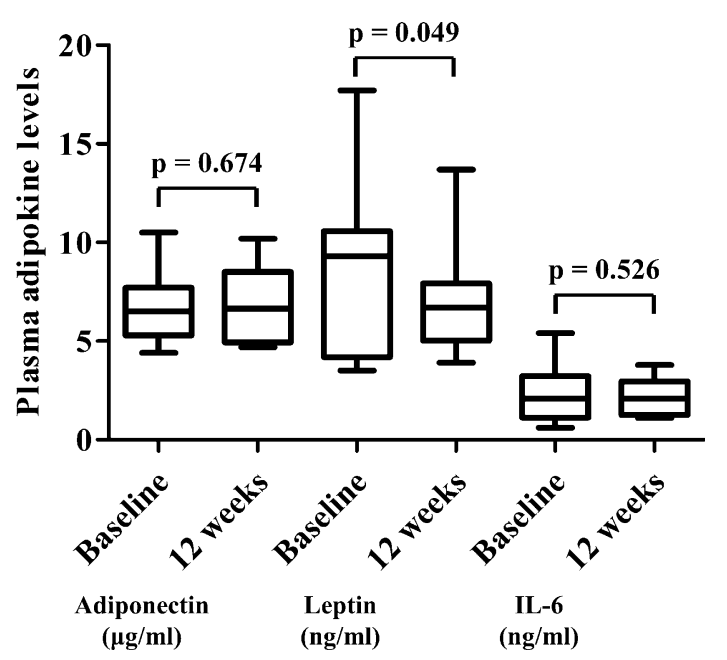

Fig. 3 Plasma adipokine levels (adiponectin, leptin, and interleukin-6 [IL-6]) at baseline and at 12 weeks after the administration of ipraglifrozin. Data are presented as the median with interquartile range

parallel with weight reduction in type 2 diabetic patients with obesity [21]. However, these results were obtained from obese patients, and little has been reported regarding the preferable effects of diabetic medications on the EF in non-obese patients with diabetes. To our knowledge, we showed for the first time the effects of ipraglifrozin on the EF in non-obese patients with type 2 diabetes. Thus, SGLT2 inhibitors may be involved in the therapeutic 
Table 4 Correlations of the changes of epicardial fat volume with markers for body composition and the markers for cardiometabolic risks in patients with type 2 diabetes

\begin{tabular}{|c|c|c|}
\hline & $r$ & $p$ values \\
\hline Body weight (kg) & 0.710 & 0.032 \\
\hline Body mass index $\left(\mathrm{kg} / \mathrm{m}^{2}\right)$ & 0.740 & 0.023 \\
\hline Liver attenuation index & 0.233 & 0.546 \\
\hline Visceral fat are $\left(\mathrm{cm}^{2}\right)$ & 0.467 & 0.205 \\
\hline Subcutaneous fat area $\left(\mathrm{cm}^{2}\right)$ & 0.733 & 0.025 \\
\hline Body fat (\%) & 0.213 & 0.582 \\
\hline Total fat mass $(\mathrm{kg})$ & 0.260 & 0.500 \\
\hline Total non-fat mass (kg) & 0.477 & 0.195 \\
\hline Skeletal muscle index & 0.342 & 0.368 \\
\hline HbAlc $(\%)$ & 0.341 & 0.370 \\
\hline HOMA-IR & -0.383 & 0.308 \\
\hline Triglycerides (mmol/l) & -0.067 & 0.865 \\
\hline HDL cholesterol (mmol/l) & -0.395 & 0.292 \\
\hline $\operatorname{ALT}(\mathrm{U} / \mathrm{l})$ & -0.372 & 0.325 \\
\hline Log C-reactive protein $(\mathrm{mg} / \mathrm{l})$ & -0.435 & 0.242 \\
\hline $\log$ ACR (mg/g) & 0.233 & 0.546 \\
\hline Adiponectin $(\mu \mathrm{g} / \mathrm{ml})$ & 0.071 & 0.867 \\
\hline Leptin $(\mathrm{ng} / \mathrm{ml})$ & -0.405 & 0.320 \\
\hline Log interleukin $6(\mathrm{pg} / \mathrm{ml})$ & -0.262 & 0.531 \\
\hline
\end{tabular}

$A C R$ albumin-to-creatinine ratio, $A L T$ alanine transaminase, $H D L$ high-density lipoprotein, HOMA-IR homeostasis model assessment as an index of insulin resistance

approach of diabetes aimed at the reduction of EFV even in patients without obesity.

In the present study, we also revealed that the administration of ipraglifrozin reduces the leptin level (Fig. 3). Leptin is secreted by adipocytes and regulates both food intake and energy expenditure [35]. It is well known that serum level of leptin was significantly and positively correlated with BMI and percentage of body fat [36]. In our research, we demonstrated that the administration of ipraglifrozin reduces the body weight and BMI (Table 1), leading to the reduction in leptin. The linear relationship between leptin and BMI level was observed even in non-obese people [37]. Meanwhile some studies reported that increased leptin level was associated with cardiometabolic risks and atherosclerosis independently of BMI. Reilly et al. reported that plasma leptin level is associated with coronary artery calcification after adjustment for BMI in patients with type 2 diabetes [38]. A recent meta-analysis also showed that the level of leptin is significantly associated with cardiometabolic risks including high blood pressure and dyslipidemia even after adjustment for the BMI [39]. These findings indicate that the reduction in level of leptin by ipraglifrozin may contribute to the prevention of future CVD in non-obese patents as well as EF.

Regarding the correlations between the changes of the markers for body composition and cardiometabolic risks and $\mathrm{EF}$, the changes of body weight, BMI, and SFA were significantly correlated with that of EF (Table 4). Declines in body weight and BMI were reported to be associated with the decline in EF in severely obese subjects $[10,40]$. SFA was also reported to be decreased in parallel with the decline in body weight in patients receiving bariatric surgery [41]. Our results (Table 4) are consistent with these reports. In addition to body weight, BMI, and SFA, several studies showed that VFA was strongly correlated with EFV [42, 43]. However, Gaborit et al. demonstrated that the decline in EFV is not associated with that in VFA, and the authors also reported the possibility that sleep apnea syndrome impaired the reduction of EFV in patients receiving bariatric surgery [44]. Consistently with the report by Gaborit et al., we did not observe a significant correlation between the change of EFV and that of VFA.

In contrast to the favorable effects of ipraglifrozin, the reduction of lean mass including skeletal muscle mass was accompanied by the reduction of weight caused by the administration of SGLT2 inhibitors [45]. The reduction of skeletal muscle mass is a component of sarcopenia which is defined as the progressive loss of muscle mass and function with aging $[46,47]$. Other than aging, diabetic patients are also reported to be at a high risk of sarcopenia [48]. In addition, low BMI is a strong predictor 
of reduced skeletal muscle mass [49]. These previous studies suggest that the administration of SGLT2 inhibitors should be carefully considered in non-obese patients with type 2 diabetes, although SMI was not decreased by the ipraglifrozin treatment in our study.

The strength of our study is that the EFV was measured using MRI which is recognized as the gold standard modality for imaging EFV because of the measurement accuracy [9]. Potential limitations of our study include the study design (single-arm), small sample size, no information on the diet and exercise, and ethnically homogenous population (Japanese). In addition, although diet, exercise, or some antidiabetic medications remained unchanged during the participation period of this study, these were reported to affect the epicardial fat accumulation as well as body weight and could be confounding factors in the association between treatment of ipragliflozin and reduction of epicardial fat due to the single-arm design. Further large randomized controlled trials are needed to confirm the impact of ipragliflozin or SGLT2 inhibitors for the reduction of epicardial fat in non-obese type 2 diabetic patients.

\section{CONCLUSIONS}

We demonstrate that EFV and leptin can be reduced by the treatment of ipraglifrozin which is accompanied with the reduction of weight and with the improvement of glycemic control, lipid profile, and insulin resistance in non-obese Japanese patients with type 2 diabetes.

\section{ACKNOWLEDGEMENTS}

This study was sponsored by Grants-in-Aid for Scientific Research from the Ministry of Education, Culture, Sports, Science and Technology of Japan and Astellas Pharma Inc. The study sponsors also funded article processing charges. All authors had full access to all of the data in this study and take complete responsibility for the integrity of the data and accuracy of the data analysis. All named authors meet the International Committee of Medical
Journal Editors (ICMJE) criteria for authorship for this manuscript, take responsibility for the integrity of the work as a whole, and have given final approval for the version to be published.

Disclosures. Tatsuya Fukuda, Ryotaro Bouchi, Masahiro Terashima, Yuriko Sasahara, Masahiro Asakawa, Takato Takeuchi, Yujiro Nakano, Masanori Murakami, Isao Minami, Hajime Izumiyama, Koshi Hashimoto, Takanobu Yoshimoto, and Yoshihiro Ogawa declare that they have nothing to disclose.

Compliance with Ethics Guidelines. The study was approved by the Institutional Review Board (IRB) at Tokyo Medical and Dental University (No. 2102). All procedures followed were in accordance with the ethical standards of the responsible committee on human experimentation (institutional and national) and with the Helsinki Declaration of 1964, as revised in 2013. Informed consent was obtained from all patients for being included in the study.

Data Availability. The datasets analyzed for the current analysis are available from the corresponding author on reasonable request.

Open Access. This article is distributed under the terms of the Creative Commons Attribution-NonCommercial 4.0 International License (http://creativecommons.org/licenses/ by-nc/4.0/), which permits any noncommercial use, distribution, and reproduction in any medium, provided you give appropriate credit to the original author(s) and the source, provide a link to the Creative Commons license, and indicate if changes were made.

\section{REFERENCES}

1. Rexrode KM, Manson JE, Hennekens CH. Obesity and cardiovascular disease. Curr Opin Cardiol. 1996;11:490-5.

2. Mokdad AH, Ford ES, Bowman BA, et al. Prevalence of obesity, diabetes, and obesity-related health risk factors, 2001. JAMA. 2003;289:76-9. 
3. Kahn BB, Flier JS. Obesity and insulin resistance. J Clin Invest. 2000;106:473-81.

4. Després JP, Lemieux S, Lamarche B, et al. The insulin resistance-dyslipidemic syndrome: contribution of visceral obesity and therapeutic implications. Int J Obes Relat Metab Disord. 1995;19(Suppl 1):S76-86.

5. Fox CS, Massaro JM, Hoffmann U, et al. Abdominal visceral and subcutaneous adipose tissue compartments: association with metabolic risk factors in the Framingham Heart Study. Circulation. 2007;116:39-48.

6. Bouchi $\mathrm{R}$, Minami I, Ohara $\mathrm{N}$, et al. Impact of increased visceral adiposity with normal weight on the progression of arterial stiffness in Japanese patients with type 2 diabetes. BMJ Open Diabetes Res Care. 2015;3:e000081.

7. Sacks HS, Fain JN. Human epicardial adipose tissue: a review. Am Heart J. 2007;153:907-17.

8. Lim S, Meigs JB. Ectopic fat and cardiometabolic and vascular risk. Int J Cardiol. 2013;169:166-76.

9. Bertaso AG, Bertol D, Duncan BB, Foppa M. Epicardial fat: definition, measurements and systematic review of main outcomes. Arq Bras Cardiol. 2013;101:e18-28.

10. Willens HJ, Byers P, Chirinos JA, Labrador E, Hare JM, de Marchena E. Effects of weight loss after bariatric surgery on epicardial fat measured using echocardiography. Am J Cardiol. 2007;99:1242-5.

11. Iacobellis G, Leonetti F. Epicardial adipose tissue and insulin resistance in obese subjects. J Clin Endocrinol Metab. 2005;90:6300-2.

12. Rijzewijk LJ, Jonker JT, van der Meer RW, et al. Effects of hepatic triglyceride content on myocardial metabolism in type 2 diabetes. J Am Coll Cardiol. 2010;56:225-33.

13. Mahabadi AA, Berg MH, Lehmann N, et al. Association of epicardial fat with cardiovascular risk factors and incident myocardial infarction in the general population: the Heinz Nixdorf Recall Study. J Am Coll Cardiol. 2013;61:1388-95.

14. Okada $\mathrm{K}$, Ohshima $\mathrm{S}$, Isobe $\mathrm{S}$, et al. Epicardial fat volume correlates with severity of coronary artery disease in nonobese patients. J Cardiovasc Med (Hagerstown). 2014;15:384-90.

15. Yong HS, Kim EJ, Seo HS, et al. Pericardial fat is more abundant in patients with coronary atherosclerosis and even in the non-obese patients: evaluation with cardiac CT angiography. Int J Cardiovasc Imaging. 2010;26(Suppl 1):53-62.
16. Zinman B, Wanner C, Lachin JM, et al. Empagliflozin, cardiovascular outcomes, and mortality in type 2 diabetes. N Engl J Med. 2015;373:2117-28.

17. Ridderstråle $\mathrm{M}$, Andersen $\mathrm{KR}$, Zeller C, et al. Comparison of empagliflozin and glimepiride as add-on to metformin in patients with type 2 diabetes: a 104-week randomised, active-controlled, double-blind, phase 3 trial. Lancet Diabetes Endocrinol. 2014;2:691-700.

18. Bolinder J, Ljunggren Ö, Kullberg J, et al. Effects of dapagliflozin on body weight, total fat mass, and regional adipose tissue distribution in patients with type 2 diabetes mellitus with inadequate glycemic control on metformin. J Clin Endocrinol Metab. 2012;97:1020-31.

19. Vallon V, Gerasimova M, Rose MA, et al. SGLT2 inhibitor empagliflozin reduces renal growth and albuminuria in proportion to hyperglycemia and prevents glomerular hyperfiltration in diabetic Akita mice. Am J Physiol Renal Physiol. 2014;306:F194-204.

20. Merovci A, Solis-Herrera C, Daniele G, et al. Dapagliflozin improves muscle insulin sensitivity but enhances endogenous glucose production. J Clin Invest. 2014;124:509-14.

21. Bouchi R, Terashima M, Sasahara Y, et al. Luseogliflozin reduces epicardial fat accumulation in patients with type 2 diabetes: a pilot study. Cardiovasc Diabetol. 2017;16:32.

22. Kashiwagi A, Yoshida S, Nakamura I, et al. Efficacy and safety of ipragliflozin in Japanese patients with type 2 diabetes stratified by body mass index: a subgroup analysis of five randomized clinical trials. J Diabetes Investig. 2016;7:544-54.

23. Kuzuya T, Nakagawa S, Satoh J. Report of the committee on the classification and diagnostic criteria of diabetes mellitus. J Diabetes Investig. 2010;1:212-28.

24. Matsuo S, Imai E, Horio M, et al. Revised equations for estimated GFR from serum creatinine in Japan. Am J Kidney Dis. 2009;53:982-92.

25. Bouchi R, Nakano Y, Ohara N, et al. Clinical relevance of dual-energy X-ray absorptiometry (DXA) as a simultaneous evaluation of fatty liver disease and atherosclerosis in patients with type 2 diabetes. Cardiovasc Diabetol. 2016;15:64.

26. Bouchi $\mathrm{R}$, Takeuchi $\mathrm{T}$, Akihisa $\mathrm{M}$, et al. Increased visceral adiposity with normal weight is associated with prevalence of non-alcoholic fatty liver disease in Japanese patients with type 2 diabetes. J Diabetes Investig. 2016;7:607-14. 
27. Nagata M, Kato S, Kitagawa $K$, et al. Diagnostic accuracy of 1.5-T unenhanced whole-heart coronary MR angiography performed with 32-channel cardiac coils: initial single-center experience. Radiology. 2011;259:384-92.

28. Alexopoulos N, McLean DS, Janik M, Arepalli CD, Stillman AE, Raggi P. Epicardial adipose tissue and coronary artery plaque characteristics. Atherosclerosis. 2010;210:150-4.

29. Oka T, Yamamoto H, Ohashi N, et al. Association between epicardial adipose tissue volume and characteristics of non-calcified plaques assessed by coronary computed tomographic angiography. Int J Cardiol. 2012;161:45-9.

30. Levelt E, Pavlides M, Banerjee R, et al. Ectopic and visceral fat deposition in lean and obese patients with type 2 diabetes. J Am Coll Cardiol. 2016;68:53-63.

31. Kim MK, Tomita T, Kim MJ, Sasai H, Maeda S, Tanaka K. Aerobic exercise training reduces epicardial fat in obese men. J Appl Physiol. 1985;2009(106):5-11.

32. Sacks HS, Fain JN, Cheema P, et al. Inflammatory genes in epicardial fat contiguous with coronary atherosclerosis in the metabolic syndrome and type 2 diabetes: changes associated with pioglitazone. Diabetes Care. 2011;34:730-3.

33. Lima-Martínez MM, Paoli M, Rodney M, et al. Effect of sitagliptin on epicardial fat thickness in subjects with type 2 diabetes and obesity: a pilot study. Endocrine. 2016;51:448-55.

34. Iacobellis $\mathrm{G}$, Mohseni $\mathrm{M}$, Bianco $\mathrm{S}$. Liraglutide causes massive and rapid reduction of cardiac fat independent of weight loss in type 2 diabetes. In: The 75th American Diabetes Association, Scientific Sessions, LBA-5785, Boston, Mass, USA, 2015.

35. Friedman J. 20 years of leptin: leptin at 20: an overview. J Endocrinol. 2014;23:1-8.

36. Considine RV, Sinha MK, Heiman ML, et al. Serum immunoreactive-leptin concentrations in normal-weight and obese humans. $\mathrm{N}$ Engl J Med. 1996;334:292-5.

37. Kazmi A, Sattar A, Hashim R, Khan SP, Younus M, Khan FA. Serum leptin values in the healthy obese and non-obese subjects of Rawalpindi. J Pak Med Assoc. 2013;63:245-8.

38. Reilly MP, Iqbal N, Schutta M, et al. Plasma leptin levels are associated with coronary atherosclerosis in type 2 diabetes. J Clin Endocrinol Metab. 2004;89:3872-8.
39. Sattar N, Wannamethee G, Sarwar N, et al. Leptin and coronary heart disease: prospective study and systematic review. J Am Coll Cardiol. 2009;53:167-75.

40. Iacobellis G, Singh N, Wharton S, Sharma AM. Substantial changes in epicardial fat thickness after weight loss in severely obese subjects. Obesity (Silver Spring). 2008;16:1693-7.

41. Foppa M, Pond KK, Jones DB, Schneider B, Kissinger $\mathrm{KV}$, Manning WJ. Subcutaneous fat thickness, but not epicardial fat thickness, parallels weight reduction three months after bariatric surgery: a cardiac magnetic resonance study. Int $\mathrm{J}$ Cardiol. 2013;168:4532-3.

42. Iacobellis G, Ribaudo MC, Assael F, et al. Echocardiographic epicardial adipose tissue is related to anthropometric and clinical parameters of metabolic syndrome: a new indicator of cardiovascular risk. J Clin Endocrinol Metab. 2003;88:5163-8.

43. Iacobellis G, Assael F, Ribaudo MC, et al. Epicardial fat from echocardiography: a new method for visceral adipose tissue prediction. Obes Res. 2003;11:304-10.

44. Gaborit B, Jacquier A, Kober F, et al. Effects of bariatric surgery on cardiac ectopic fat: lesser decrease in epicardial fat compared to visceral fat loss and no change in myocardial triglyceride content. J Am Coll Cardiol. 2012;60:1381-9.

45. Cefalu WT, Leiter LA, Yoon KH, et al. Efficacy and safety of canagliflozin versus glimepiride in patients with type 2 diabetes inadequately controlled with metformin (CANTATA-SU): 52 week results from a randomised, double-blind, phase 3 non-inferiority trial. Lancet. 2013;382:941-50.

46. Cruz-Jentoft AJ, Baeyens JP, Bauer JM, European Working Group on Sarcopenia in Older People, et al. Sarcopenia: European consensus on definition and diagnosis: report of the European Working Group on Sarcopenia in Older People. Age Aging. 2010;39:412-23.

47. Chen LK, Liu LK, Woo J, et al. Sarcopenia in Asia: consensus report of the Asian Working Group for Sarcopenia. J Am Med Dir Assoc. 2014;15:95-101.

48. Park SW, Goodpaster BH, Lee JS, et al. Excessive loss of skeletal muscle mass in older adults with type 2 diabetes. Diabetes Care. 2009;32:1993-7.

49. Iannuzzi-Sucich M, Prestwood KM, Kenny AM. Prevalence of sarcopenia and predictors of skeletal muscle mass in healthy, older men and women. J Gerontol A Biol Sci Med Sci. 2002;57:M772-7. 